\title{
Etiology and Pathophysiology of Tinnitus - A Systematic Review
}

Sujoy Kumar Makar

\begin{abstract}
Introduction: Prevalence of tinnitus range from $7.1 \%$ to $14.6 \%$ (National Center for Health Statistics, 2016), but the mechanisms responsible for the development of this abnormal sensory state remain poorly understood.

Objectives: To determine the evidence for different etiologies and pathophysiology of tinnitus identified by clinical diagnostic tests in the adult population.

Study Design: Systematic literature review.

Methods: Review of data base using PRISMA guidelines: Google Scholar, Medline, Springer Link, Pubmed. In addition, manual reference search of identified papers. Randomized controlled trials, case control study, prospective cohort studies, and retrospective reviews of consecutive patients in which clear data were reported with respect to etiology and pathophysiology of tinnitus.

Results: Sixty seven articles met the inclusion criteria. The papers searched recent studies from 2004 to 2018 for different etiologies such as noise exposure, age, ototoxic drugs, hearing loss among patients with tinnitus. Multiple pathophysiology were identified, including inner ear pathology, auditory nerve synchronisation, central nervous system anomalies and limbic and autonomous nervous system problems. The group of papers evaluated tinnitus patients with specific diagnostic tests such as pure tone audiometry, Immitance audiometry, otoacoustic emission, Auditory brainstem response and diagnostic imaging of fMRI, MRI and PET study.

Conclusions: The results indicate a high level of heterogeneity between the studies for all the assessed areas. These results support the need for greater stratification of the tinnitus population and the importance of a standardized Puretone audiometry with extended high frequency, OAE, ABR and diagnostic imaging (fMRI, MRI \& PET) method to make comparisons between studies possible. Diagnostic imaging is an important useful method for identification of intracranial pathology that can present with tinnitus as a primary symptom. Establishment of a direct causal link between tinnitus and these etiologies and pathophysiology remains elusive.
\end{abstract}

Keywords: Tinnitus; Loudness; Etiology; pathophysiology. 


\section{INTRODUCTION}

Tinnitus is the perception of a continuous or intermittent sound in the absence of external acoustic stimulation'. According to Bhatt, Lin and Bhattacharyya ${ }^{2}$, prevalence of tinnitus in the United States is approximately 1 in 10 adults. The overall prevalence of tinnitus was $13.5 \%$ in patients aged below 50 years and $34.4 \%$ in patients aged above 67 years. The incidence was $27.8 \%$ in Sweden as reported by $(2017)^{3}$. Several risk factors for the development of tinnitus are noise (19.6\%), ototoxicity (16.8\%), presbycusis (16.3\%) and increasing age (16.3\%) as reported (2014). It is also possible to have severe tinnitus with no evidence of any aural pathology. Further, tinnitus sufferers want to know how their tinnitus is generated and whether it is curable. However, till date the literature is not able to fully explain about the pathophysiology of subjective tinnitus and cannot assure patients about the prognosis of tinnitus. It is essential to have evidence based on its underlying aetiology and pathophysiology to treat tinnitus effectively; once the aetiology and pathophysiology is known, the disorder can be treated ${ }^{4-6}$. However, in case of subjective tinnitus, it is difficult to identify a single origin of tinnitus. Hence it is difficult to treat it completely till date. Tinnitus is of interest to audiologists because it comes under their professional domain and it creates problems in human health. Therefore, it is necessary for the audiologists to understand the evidence based pathophysiology of tinnitus to explain changes of brain activity in tinnitus patients for better management and prevention of tinnitus. Review of literature is an integral part of research ${ }^{7}$. This involves identification and analysis of documents containing information related to the research problem, with the purpose of providing context for the research and its justification, identifying the areas which have been already covered as well as the research gaps. Studied a systematic review to assess the scientific evidence on the associations between symptoms of depression and tinnitus. A systematic review of tinnitus prevalence and severity. However, there is a need to study how much high-level evidence exists for the aetiology and complex pathophysiology of tinnitus. To that end, this article provides a broad-based review of what is presently known about aetiology, involvement of cochlea, auditory nerve, auditory cortex and somatosensory systems in tinnitus patients and its clinical implications. The summary of fundamental information has relevance to both clinical and research arenas. The research question is "Does high level evidence exist to support aetiology and pathophysiology of tinnitus?" The strength of present systematic review search examines etiology and pathophysiology of tinnitus as well as validated measures to find out aetiology in the auditory pathways-10.

\section{MATERIALS AND METHODS}

A systematic review of the literature was performed including searches by using PRISMA guidelines the following electronic data base: Google Scholar, Medline, Springer Link, Pubmed. The key words were: Causes of tinnitus, tinnitus, cochlear tinnitus, neural tinnitus, origin of tinnitus, neurophysiological and psycho- physical dimension of tinnitus, origin of tinnitus, cortical tinnitus, somatosensory tinnitus, neural plasticity changes in tinnitus patients, epidemiologic studies, risk factors, cohort, case control, randomized controlled trial or controlled clinical, etc. Articles were reviewed using a prior determined selection criterion. Inclusion criteria included: randomized controlled trials, prospective cohort studies, retrospective reviews. Exclusion criteria included: prospective or retrospective study, comments, practice guidelines, editorials letters, book chapters, except that references were reviewed ${ }^{11-15}$. This systemic review is a humble attempt to identify the origin and pathophysiology of tinnitus. Accessed 425 publications, and doted down to 57 original papers and 10 review papers identifying the latest procedures used to cite the origin and pathophysiology. Studies were selected that used PTA, IA, OAE, ABR, ( $f$ MRI ), ( $r$ CBF ), PET, $M E G$ for investigetion. The review gives importance to neurophysiological and psycho- physical dimension of tinnitus. Thus the composition of the processes involved in tinnitus is included in the review. Journal titles were independently reviewed, and articles were included if researcher felt they were relevant. Selected abstracts were then reviewed using the predetermined inclusion and exclusion criteria. Articles that were considered relevant or uncertain relevance were retrieved as full text articles. The full text papers were reviewed and data extraction performed independently ${ }^{16-22}$.

\section{RESULTS}

Out of the 67 studies that met the search criteria, 54 were research studies, 10 were review studies and 3 case study report (Figure 1). A descriptive summary including the type of study, research design, sample size, measurement and result are provided in Tables 1 and 2 . Several combined objective studies suggest that dysfunction starts in the cochlea and then a weak imbalance of neural activity is generated in the central pathway; this is noticed at low signal level in the auditory systems and being a new signal it is enhanced by sub-cortical centres, transferred to the auditory cortex and perceived as an abnormal sound tinnitus. Longer duration involvement of auditory system in tinnitus patients affect limbic system and autonomic nervous system also. Thus this evidence supports the viewpoint that in longer duration tinnitus there is involvement of whole brainstem; also that multiple feature tinnitus are a result of abnormal activity within the central auditory pathway ${ }^{23-25}$. These abnormalities which are due to abnormal activity in IC, CN, MSOC and brainstem, lead to changes in tonotopic organisation of auditory maps. Thus original abnormalities trigger secondary abnormalities and this could explain why pitch, loudness and $\mathrm{Rl}$ of tinnitus change in longer duration tinnitus. Cochlear-type tinnitus is suggested to result from aberrant activity generated at the cochlear nerve level. The outer hair cells regulate the endocochlear potential that contribute to enhancement of cochlear spontaneous 


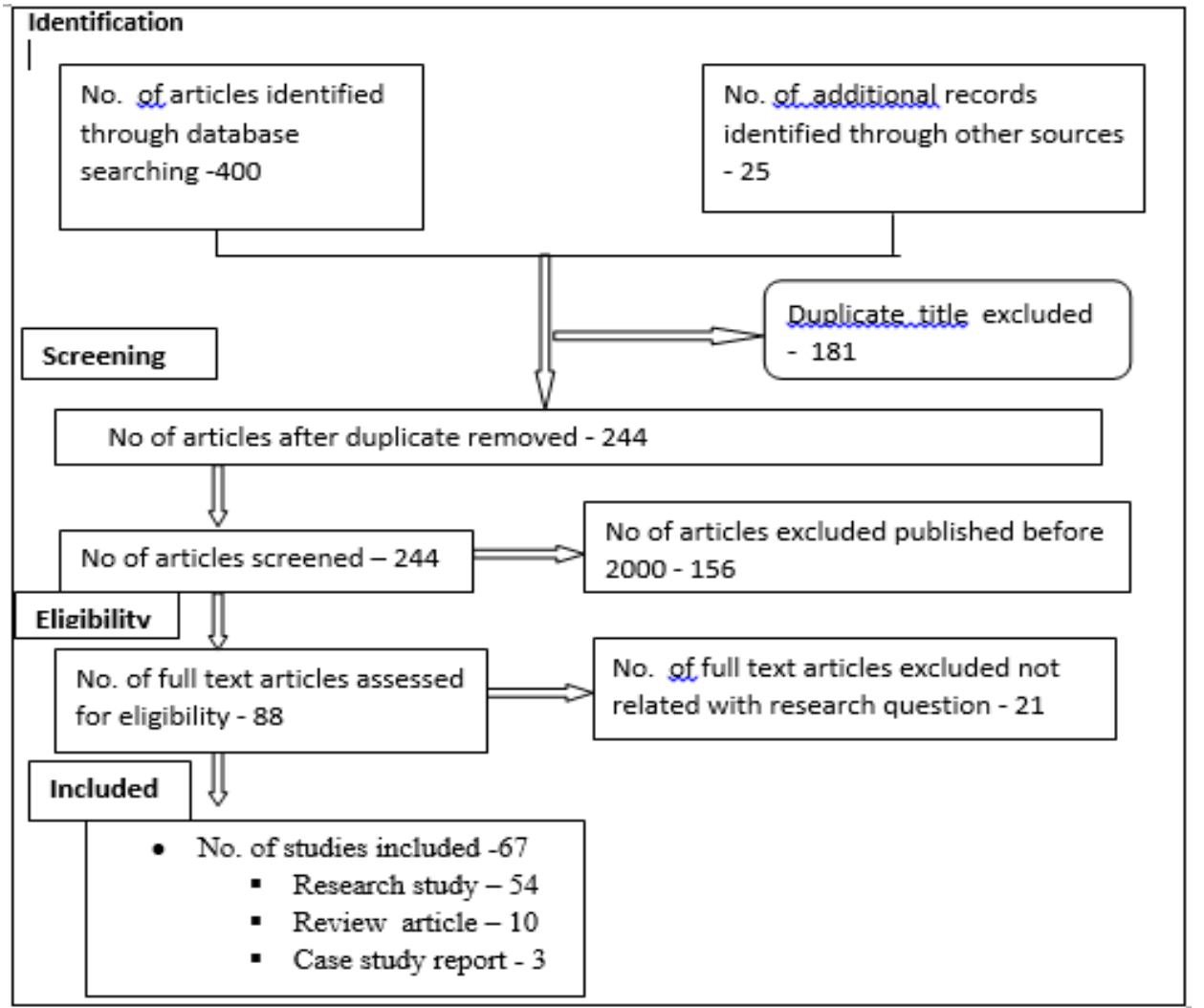

Figure 1: Systematic literature review using PRISMA guidelines step by step.

activity. A reduction in cochlear activity produces tinnitusrelated plastic changes, namely cortical reorganisation, thalamic neuron hyperpolarisation, facilitation of nonauditory limbic inputs and increase in central gain. These central changes are associated with abnormal patterns of spontaneous activity in the auditory pathway, i.e. hypersynchrony activity. The somatosensory system, and the limbic and autonomic nervous systems are also involved in tinnitus generation/manipulation ${ }^{26-30}$.

Etiology and Hearing loss: Sensorineural hearing loss is commonly accompanied with tinnitus. Some researchers believe that subjective tinnitus cannot exist without hearing loss (American Tinnitus Association, 2019). Subjective tinnitus has no identified cause other than hearing loss (ASHA, 2019) even individuals with tinnitus and normal hearing show a significant hearing loss at extended higher frequency 10,000 to $20,000 \mathrm{~Hz}$ ${ }^{31}$. Reported that there was significant differences of high frequency threshold between tinnitus ear and nontinnitus ear $(P<0.01)$; also significant differences of high frequency threshold between tinnitus ear and non-tinnitus ear in each group $(P<0.01) .{ }^{32}$ suggested that in patients with unilateral tinnitus, hearing threshold $(0.125-8 \mathrm{kHz})$ of tinnitus ear and contralateral ear difference was not statistically significant $(P<0.05)$, but in extended high frequency $(>10 \mathrm{kHz})$ the difference between two ears was statistically significant $(P>0.05)$. Shim et al (2009) reported that patients with tinnitus who have normal hearing below $8 \mathrm{kHz}$ have decreased hearing ability at extended high-frequencies at $10 \mathrm{kHz}, 12 \mathrm{kHz}, 14 \mathrm{kHz}$, and $16 \mathrm{kHz}$. statistically significant differences $(p<0.01)$ were found between the determination of the frequency of tinnitus made with conventional and high-frequency audiometers, as well as a correlation between highfrequency tinnitus and distress expressed by patients. ${ }^{33-35}$ reported that the frequency is an important factor in tinnitus group: between $8,000 \mathrm{~Hz}$ to $20,000 \mathrm{~Hz}$, with the increases in frequency, hearing loss also increase. Many studies have represented tinnitus as a threshold phenomenon for which any one factor, such as chronic progressive hearing loss is insufficient to elicit its emergence--two or more trigger factors (i.e., psychosocial stress, noise exposure, and somatic factors) can act synergistically to produce symptomatic tinnitus ${ }^{36-40}$.

Noise: According to ASHA (2019) loud noise exposure might cause tinnitus (ASHA, 2019). Population-based data indicate that excessive noise exposure represents the second most common cause of tinnitus ${ }^{41-43}$ offer a review of several studies suggested that noise trauma is the single most unique cause of tinnitus (18\%), followed by head and neck trauma (8\%), whereas drugs (most often salicylate) only account for $2 \%$ of known incidents of tinnitus. Small temporary changes in the outer hair cells (OHCs) following noise exposure can also trigger the emergence of tinnitus by increasing the gain of the central auditory system ${ }^{44}$. Noise induced hearing loss $(\mathrm{NIHL})$ is most prevalent cause of tinnitus ${ }^{45}$. Many environmental factors can cause tinnitus, mostly related to the effect of noise on the auditory system and subsequent damage to the microstructures in the cochlea ${ }^{46-48}$. According to American Tinnitus Association 2019, exposure to loud noises in a single traumatic experience or over time can 
Table 1: Summary of Etiology and Pathphygilogy of Tinnitus studies utilizing a diagnostic algorithm.

Etiology /
pathophysiology

Noise and other causes

Hearing loss and tinnitus

\section{Etiology}

Bhatt, Lin, \& Bhattacharya (2016)

Samarei \& Fatholah (2004)

Kujawa \& Liberman (2009).

Noreña \& Eggermont (2006)

Tang, Ji \& Liu (2011)

Case control study

$n=200$

Yildirim, Berkiten, Kuzdere \& Ugras (2010)

Shargorodsky, Curhan \& Farwell (2010)

Shim et al., (2009)

Cross-sectional analysis

$n=75764$

\section{Experimental} study
Experimental study

Purposive sampling $n=47$
PTA with extended high frequency

ENT, IA, PTA with high frequency
ABR, ECOGG, DPOAE, cochlear mapping software

Loud noises at work (odds ratio, 3.3; 95\%

$\mathrm{Cl}, 2.9-3.7)$ and recreational noise (odds ratio, 2.6; $95 \% \mathrm{Cl}, 2.3-2.9$ ). Years of workrelated noise exposure correlated with increasing prevalence of tinnitus $(r=0.13$; $95 \% \mathrm{Cl}, 0.10-0.16)$.

Most common causes of tinnitus were noise (19.6\%), ototoxicity (16.8\%) and presbycusis $(16.3 \%)$.

Damage to the hair cells has progressive consequences that are considerably more widespread than are revealed by conventional threshold testing. This primary neurodegeneration should add to difficulties in hearing in noisy environments, and could contribute to tinnitus, commonly associated with inner ear damage.

Noise-induced hearing loss induces reorganization of the tonotopic map in auditory cortex and increases spontaneous firing rate and neural synchrony. This is interpreted as an absence of putative neural signs of tinnitus.

Statistically significant differences reported between the determination of the frequency of tinnitus made with conventional and high-frequency audiometers, as well as a correlation between high-frequency tinnitus and distress expressed by patients.

In patients with unilateral tinnitus, the difference in hearing threshold of tinnitus ear and contralateral ear $(0.125$ to 8 $\mathrm{kHz}$ ), was statistically significant, but in extended high frequency (> $10 \mathrm{kHz}$ ), the difference between two ears was not statistically significant.

There were significant hearing loss related to age and frequency from $8,000-20,000$ $\mathrm{Hz}$ of the patients with normal hearing in

PTA, IA, OE, tinnitus pitch and loudness matching.

Case control study $\quad n=154$
250-4,000 $\mathrm{Hz}$ frequency range.

The frequency between $8,000 \mathrm{~Hz}$ to $20,000 \mathrm{~Hz}$ is an important factor in tinnitus group. As the frequency increases, hearing loss increases.

The significant association between tinnitus and age $(p<.01),(60$ to 69 years), smoking $(p<.01)$, hypertension $(p<.01)$, diabetes mellitus $(p<.01)$ suggest that vascular disease might have a greater contribution to the etiology of tinnitus.

Patients with tinnitus who have normal High frequency PTA, ABR, tinnitus pitch \& loudness hearing below $8 \mathrm{kHz}$ have decreased

hearing ability at extended high-

frequencies at $10 \mathrm{kHz}, 12 \mathrm{kHz}, 14 \mathrm{kHz}$, and $16 \mathrm{kHz}$. 


\section{Cai \& Tang · (2004) Case control study $\quad n=78$}

Sindhusake et al., (2003)

\section{Cross-sectional} study

$n=2145$
PTA, TEOAE, SOAE

PTA, IA, ABR, DPOAE, Tinnitus, pitch and loudness

Significant differences of HF threshold between tinnitus ear and non tinnitus ear.

PTA, IA, tinnitus pitch and loudness.

Also significant differences of $\mathrm{HF}$ threshold between ipsilateral ear (tinnitus ear) and that of contralateral ear (no tinnitus ear) in each group.

Risk of tinnitus were poorer hearing and cochlear function, self-reported workrelated noise exposure, and history of middle ear or sinus infections, severe neck injury or migraine

DPOAE results suggested cochlear involvement and $A B R$ findings indicate whole brainstem function is involved in longer duration \& multiple features tinnitus.

Table 2: Charting the data of systematic review for studies.

\begin{tabular}{|c|c|c|c|}
\hline $\begin{array}{l}\text { Authors } \\
\text { and Year }\end{array}$ & Title & Methods & Results \\
\hline $\begin{array}{l}\text { Henry, Roberts, Caspary, } \\
\text { Theodoroff \& } \\
\text { Salvi (2014) }\end{array}$ & $\begin{array}{l}\text { Underlying Mechanisms of } \\
\text { Tinnitus: Review \& Clinical } \\
\quad \text { Implications }\end{array}$ & Review & $\begin{array}{l}\text { Tinnitus is a pathology involving neuroplastic changes in central } \\
\text { auditory structures that take place when brain is deprived of its } \\
\text { normal input by pathology in the cochlea. }\end{array}$ \\
\hline $\begin{array}{l}\text { Lockwood, } \\
\text { Salvi \& Burkad (2002) }\end{array}$ & Tinnitus & Review & $\begin{array}{l}\text { Cochlear damage leads to a reorganization of the pathways in the } \\
\text { central auditory system. Reduction in auditory nerve input leads to } \\
\text { disinhibition of the DCN and an increase in spontaneous activity in } \\
\text { the central auditory system, which is experienced as tinnitus. }\end{array}$ \\
\hline $\begin{array}{l}\text { Rauschecker, J. P., Leaver, A. } \\
\text { M., \& Mühlau, M. (2010). }\end{array}$ & $\begin{array}{l}\text { Tuning Out the Noise: Limbic- } \\
\text { Auditory Interactions in } \\
\text { Tinnitus }\end{array}$ & Review & $\begin{array}{l}\text { Loss of input in the lower lesion frequency range in cochlea leads to } \\
\text { an over representation of lesion-edge frequencies, which causes } \\
\text { hyperactivity and possible burst-firing in central auditory pathways, } \\
\text { constituting the initial tinnitus signal. Under normal circumstances, } \\
\text { the tinnitus signal is cancelled out at the level of the thalamus } \\
\text { by an inhibitory feedback loop originating in paralimbic structures, } \\
\text { If the limbic regions are compromised, this "noise-cancellation" } \\
\text { mechanism breaks down, and chronic tinnitus results. }\end{array}$ \\
\hline $\begin{array}{l}\text { Han, Lee, Kim, Lim, \& } \\
\text { Shin (2009) }\end{array}$ & $\begin{array}{l}\text { Tinnitus: Characteristics, } \\
\text { Causes, Mechanisms, and } \\
\text { Treatments }\end{array}$ & Review & $\begin{array}{l}\text { Tinnitus generators are theoretically located in the auditory pathway, } \\
\text { and such generators and various mechanisms occurring in the } \\
\text { peripheral auditory system and central auditory system have been } \\
\text { explained in terms of the auditory plasticity theory, the crosstalk } \\
\text { theory, the somatosensory, limbic and autonomic nervous systems. }\end{array}$ \\
\hline $\begin{array}{l}\text { Noreña } \\
(2015)\end{array}$ & $\begin{array}{l}\text { Revisiting the Cochlear } \\
\text { and Central Mechanisms } \\
\text { of Tinnitus and Therapeutic } \\
\text { Approaches }\end{array}$ & Review & $\begin{array}{l}\text { Cochlear-type tinnitus is suggested to result from aberrant activity } \\
\text { generated at the cochlear nerve level. The outer hair cells regulate } \\
\text { the endocochlear potential that contribute to enhancement of } \\
\text { cochlear spontaneous activity. A reduction in cochlear activity leads } \\
\text { to tinnitus-related plastic changes, namely cortical reorganisation, } \\
\text { thalamic neuron hyperpolarisation, facilitation of non-auditory } \\
\text { inputs and/or increase in central gain. These central changes are } \\
\text { associated with abnormal patterns of spontaneous activity in the } \\
\text { auditory pathway, i.e. hypersynchrony activity. }\end{array}$ \\
\hline & & & $\begin{array}{l}\text { The neuroimaging methods } \mathrm{fMRI} \text { and PET measure signals that } \\
\text { presumably reflect the firing rates of multiple neurons and are } \\
\text { assumed to be sensitive to changes in the level of neural activity. }\end{array}$ \\
\hline $\begin{array}{l}\text { Lanting, De Kleine } \\
\text {, Van Dijik (2009) }\end{array}$ & $\begin{array}{l}\text { Neural activity underlying } \\
\text { tinnitus generation: Results } \\
\text { from PET and fMRI }\end{array}$ & Review & $\begin{array}{l}\text { The general trend emerging from the neuroimaging studies, is that } \\
\text { tinnitus in humans may correspond to enhanced neural activity } \\
\text { across several centers of the central auditory system. Non-auditory } \\
\text { areas including the frontal areas, the limbic system and the } \\
\text { cerebellum seems associated with the perception of tinnitus. }\end{array}$ \\
\hline $\begin{array}{l}\text { Milloy, Fournier, } \\
\text { Benoit, Noreña, \& Koravand } \\
\text { (2017). }\end{array}$ & $\begin{array}{l}\text { Auditory Brainstem } \\
\text { Responses in } \\
\text { Tinnitus: A Review of Who, } \\
\text { How, and What? }\end{array}$ & Review & $\begin{array}{l}\text { ABR findings suggest that the longer latency and reduced amplitude } \\
\text { of wave I for the tinnitus group with normal hearing compared to } \\
\text { matched controls was the most consistent finding across studies. }\end{array}$ \\
\hline Kaltenbach (2011). & $\begin{array}{l}\text { Tinnitus: Models and } \\
\text { mechanisms }\end{array}$ & Review & $\begin{array}{l}\text { Tinnitus stemming from imbalances in the excitatory and inhibitory } \\
\text { inputs to auditory neurons. Such changes occur at multiple levels } \\
\text { of the auditory system and involve a combination of interacting } \\
\text { phenomena that are triggered by loss of normal input from the inner } \\
\text { ear. This loss sets in motion a number of plastic readjustments in the } \\
\text { higher level central auditory system. }\end{array}$ \\
\hline
\end{tabular}


damage the auditory system and result in hearing loss and sometimes, tinnitus as well.

Age: Any pathologic lesion in the auditory pathway or any reduction in auditory nerve function due to ageing has the potential to produce tinnitus ${ }^{49}$. Bilateral subjective tinnitus can be associated with presbycusis ${ }^{50}$. The significant association between tinnitus and old age (60 to 69 years) also suggest that vascular disease might have a greater contribution to the etiology of tinnitus ${ }^{51}$. Net down regulation of functional inhibition may result from production of plastic maladaptive compensatory changes due to partial deafferentation of the central auditory system caused due to aging ${ }^{52}$. In old age hearing often deteriorates, typically starting around the age of 60 . This form of hearing loss tends to be in both ears and involves the sensory loss of high-frequency sounds. Age-related hearing loss explains, in part, why tinnitus is so prevalent among seniors (American Tinnitus Association, 2019).

Psychological Status: Excessive stress might cause tinnitus according to ASHA (2019) about $75 \%$ of new cases reporting to tinnitus clinics are related to emotional stress as the trigger factor. A Study on the risk factors for developing tinnitus reported in population based studies including psychological status of the affected individuals, suggested there are clear association between the psychological state of the individual and tinnitus; in particular, the condition is more often experienced by depressive patients (19\% vs. $39.5 \%$ of depressive patients). Tinnitus is more often associated with hearing disorders (20\% vs. $30-37 \%$ ). These data are based on a study including over 14,000 respondents with an average tinnitus prevalence of $25.3 \%$ in the individual subgroups 53. The significant association between tinnitus and hypertension $(p<.01)$ suggest that vascular disease might have a greater contribution to the etiology of tinnitus ${ }^{54}$. Reported the changes in the levels of neurosteroids in the central nervous system associated with depression could be a leading cause of tinnitus. In their conclusion, American Tinnitus Association (2019) suggested psychiatric disorders such as depression, anxiety and stress are trigger factors for tinnitus ${ }^{55}$.

Ototoxicity: Bilateral subjective tinnitus can be associated with ototoxicity ${ }^{56}$, side-effect of some oral medications, such as salicylates, nonsteroidal anti-inflammatory drugs, aminoglycoside antibiotics, loop diuretics, and chemotherapy agents ${ }^{57}$. Tinnitus is a potential short-lived side-effect of many prescription medications and if the patient stops taking the medication, the tinnitus symptoms typically receded. There are some ototoxic drugs known to cause more permanent tinnitus symptoms, such as, non-Steroidal Anti-Inflammatory Drugs (NSAIDs), certain antibiotics, cancer medications, Water pills and diuretics, Quinine-based medications (American Tinnitus Association, 2019).

Medical problem: Neurologic causes include head injury, multiple sclerosis, vestibular schwannoma and cerebellopontine- angle tumors. Infectious causes include otitis media, meningitis, syphilis, and other infections that affect hearing Tinnitus is a symptom of medical conditions such as, metabolic disorders, hypothyroidism, anemia, autoimmune disorders, lyme disease, fibromyalgia, blood vessel disorders, high blood pressure, atherosclerosis; traumatic brain injury caused by concussive shock, could damage the brain's auditory processing areas and generate tinnitus symptoms. Vestibular disorders such as acoustic neuroma, vestibular schwannoma and other tumorous growths (American Tinnitus Association, 2019) are associated with tinnitus. Recently ASHA (2019) reported that Ménière's disease, migraines, head injury, drugs or medicines that are toxic could also be linked to tinnitus. Shargorodsky, reported a significant association between tinnitus and diabetes mellitus $(p<01)$ suggestive of vascular disease having a greater contribution to the cause of tinnitus.

Somatosensory cause: Several researchers attempted to study the connection between auditory and somatosensory system, and reported modification in the loudness and pitch of tinnitus via somatic maneuvers such as jaw clenching or tensing their neck muscles both the firing rates and temporal response patterns to the sound can be modulated by trigeminal stimulation preceded by an acoustic stimulus. This bimodal integration is replicated in neurons of the IC and this receives converging inputs from both the DCN and somatosensory nuclei. Another example of somatic tinnitus is that damage to the muscles, ligaments, or cartilage in the temper mandibular joint disorder (TMJ), which shares some ligaments and nerve connections with middle ear. In many scenarios, fixing the TMJ disorder will alleviate tinnitus symptoms (American Tinnitus Association, 2019).

\section{PATHOPHYSIOLOGY}

The pathophysiology of subjective tinnitus is poorly understood so are the neuroplastic changes in central auditory structures that take place when brain is deprived of its normal input by pathology in the cochlea

Cochlear pathophysiology: According to $\mathrm{Job}^{58}$, there are evidence of cochlear outer hair cell dysfunctions in participants susceptible to tinnitus due to noise. In almost all situations $\mathrm{OHCs}$ are damaged more than IHCs, which results in the disinhibition of neurons in the dorsal cochlear nuclei (DCNs)). Therefore, there will be an area within organ of corti where OHCs are affected but IHCs are intact. This would affect coupling between the tectorial membrane and the basilar membrane, to the extent that the tectorial membrane might directly impinge upon the cilia of the IHCs, thus causing them to depolarise. The role that increased neural activity in the auditory periphery may have in tinnitus generation can be explained. When OHCs damage and IHC normal functioning, cells in the dorsal cochlear nuclei (DCNs) show increased spontaneous activity because of IHC normal input but decrease OHCs input. This spontaneous activity is perceived as tinnitus. The OHCs normally recover within a few days, but this can be delayed for up to a few months. Therefore, it is 
hypothesized that tinnitus represents a consequence of a central gain adaptation mechanism when the auditory system is confronted with a hearing loss (Two types of tinnitus have been identified; tonal and complex. Tonal tinnitus results from discordant dysfunction of $\mathrm{OHCs}$ and IHCs manifesting in a single area, whereas complex tinnitus results from multiple areas of discordance (Jastreboff, 2004). However, when patients clearly have the central type of tinnitus, such as after transaction of the auditory nerve, the $\mathrm{OHC}$ concept is not applicable and alternative mechanisms need to be considered ${ }^{58}$. Auditory and Vestibular Nerve pathophysiology: studied 75 patients age ranging from 20 to 45 years, to evaluate electrophysiologically the auditory nerve and the auditory brainstem function of patients with tinnitus and normalhearing thresholds using the auditory brainstem response (ABR). Abnormal results were found in $43 \%$ in at least 1 of the 8 parameters evaluated. Although within normal limits, the tinnitus group presented with a significant prolongation of the latencies of wave I, III, and V. The interpeak I-III, and I-V values were within the normal limits, whereas the interpeak III-V value was significantly $(p=.003)$ enlarged in the experimental group. The $V / I$ amplitude was within normal limits; however, a significant $(p=.004)$ difference was present between the two groups. The interaural latency difference of wave $V$ did not show significant differences. The study suggests even though most parameters were within normal limits that there are changes in the central pathways in the tinnitus group. Schaette and McAlpine (2011) reported tinnitus is triggered by cochlear damage, however, many tinnitus patient's audiogram are normal. They explain, in tinnitus patients with normal audiogram, ABR show significantly reduced amplitude of the wave I potential but normal amplitudes of the more centrally generated wave $\mathrm{V}$. This is direct evidence of "hidden hearing loss" that manifests as reduced neural output from the cochlea and consequent renormalization of neuronal response magnitude within the brainstem ${ }^{59}$ included 43 unilateral tinnitus patients (19 males, 24 females) and 18 control participants with normal hearing thresholds in their study. The amplitudes of wave $I$ and $V$ were measured at $90 \mathrm{~dB} n \mathrm{~nL}$ and UCLs at $500 \mathrm{~Hz}$ and $3000 \mathrm{~Hz}$ pure tones in each TE and NTE were assessed. The within-patients comparison between TEs and NTEs showed no significant differences in wave $\mathrm{I}$ and wave $\mathrm{V}$ amplitude but individual data revealed increased $\mathrm{V} / \mathrm{I}$ amplitude ratios ( mean $\pm 2 \mathrm{SD}$ ) in 3 TEs in the experimental ears. No significant differences in UCL at $500 \mathrm{~Hz}$ and $3000 \mathrm{~Hz}$ between the TEs and NTEs were found but were lower than that of control group. ABR results does not represent meaningful evidence, however, reduced sound level tolerance in both TEs and NTEs might replicate increased central gain following hidden synaptopathy that was subsequently balanced between the ears by lateral olivocochlear efferents. Conducted a study with twenty six participants the aim to evaluate existence of any association between tinnitus loudness/ onset duration and audiological profile to explain differences in prognosis. The significant differences were found in extended high frequency for pure-tone audiometry hearing thresholds and tinnitus loudness/ onset duration into tinnitus and nontinnitus ears. These are associated with an increase in tinnitus loudness and its onset duration. Nerve compression may cause artificial synapses to be formed between nerve fibres of the cochlear and vestibular nerve (crosstalk), this may occur when auditory nerve fibers are intact and some other cranial nerves are damaged. This may result in the phase-locking of the spontaneous activity of groups of auditory neurons. The breakdown of the myelin insulation of the nerve fibers may further enhance coupling. In the absence of any external sounds, it creates a neural pattern that resembles the patterns evoked by actual sounds. These cranial nerves are sensitive to compression at the root entry zone, where they are covered by myelin. This notion is applied to the cochlear-vestibular nerve, which is myelinated, and is vulnerable to compression from blood vessels or tumors impinging upon the nerve (e.g., vestibular schwannoma). This might lead to tinnitus if synchronization of the stochastic firing in the cochlear nerve is perceived as sound, as well as to cross talk synapses and tinnitus development, a process seen with vestibular schwannoma or neurovascular conflict 60 .

Dorsal Cochlear Nucleus pathophysiology: The Dorsal Cochlear Nucleus (DCN) has been implicated as a possible site for the generation of tinnitus-related signals owing to its tendency to become hyperactive following exposure to tinnitus-inducing agents such as intense sound and cisplatin. However, OHC damage triggers plastic readjustments in the $D C N$, resulting in $D C N$ fusiform cells become spontaneously hyperactive a reduction in auditory nerve input leads to elevated DCN fusiform output that act as a trigger for tinnitus-related neural activity rostral to the cochlear nucleus and an increase in spontaneous activity in the central auditory system, which manifests as tinnitus. This mechanism could explain the temporary ringing sensation that can follow exposure to loud sound the plastic readjustments in the dorsal cochlear nucleolus are slow and lead to tinnitus with a delayed onset. IHC damage prevents hyperactivity in the DCN, damage to the cochlea enhances neural activity in the central auditory pathway. Auditory plasticity emerges as a consequence of the aberrant pathway, and tinnitus might be considered as an auditory system analog to phantom limb sensations in amputees studied auditory nerve and brainstem function in response to sound assessed via auditory brainstem responses (ABR) in humans with and without tinnitus. Tinnitus participants showed reduced wave I amplitude but enhanced wave $V$ suggestive of reduced auditory nerve activity and elevated input to the inferior follicular, compared with non-tinnitus participants. It was concluded that the elevated III/I and V/I amplitude ratios in tinnitus participants reflect disproportionately high activity in the spherical bushy cells (SBC) pathway for a given amount of peripheral input. The results imply a role for the VCN in tinnitus and suggest the SBC pathway as a target for tinnitus treatment. 
Auditory cortex pathophysiology: it reported that tinnitus might be generated in the temporal lobe in the auditory association cortex and inferior colliculus on low-frequency fluctuations of $\mathrm{FMRI}$ confirms that chronic tinnitus patients have aberrant significant increased spontaneous neuronal activity within the right middle temporal gyrus (MTG), right superior frontal gyrus (SFG) and right angular gyrus. On the other hand, decreased spontaneous neuronal activity was detected in the right middle occipital gyrus and bilateral thalamus. The tinnitus duration (longer vs shorter) correlated positively with higher spontaneous neuronal activity values in right superior frontal gyrus (SFG). Using magnetic source imaging test (MRI), a marked shift of the auditory cortical representation of the tinnitus frequency into an area adjacent to the expected tonotopic location was observed. Also a strong positive correlation was found between the subjective loudness of the tinnitus and the amount of cortical reorganization $(r=0.82, p<0.01)$. Another reported that frequency region corresponding to the tinnitus pitch is known to be abnormally represented in auditory cortex. This appears to be correlated with the perceived loudness of tinnitus but not with the amount of hearing loss, which is the primary determinant of changes in tonotopic maps these results strongly demonstrate that tinnitus is related to plastic alterations in auditory cortex.

Somatosensory pathophysiology: the activity of the Dorsal Cochlear Nucleus (DCN) is also influenced by stimulation of nonauditory structures. However, the somatosensory system is the only nonauditory sensory system that appears to be related to tinnitus (e.g., temporomandibular-joint). Somatic tinnitus can develop from activation of underlying oto-somatic interaction (Levin, 2004) and is caused by disinhibition of the ipsilateral DCN; this is mediated by nerve fibers whose cell bodies lie in the ipsilateral medullary somatosensory nuclei. These neurons receive inputs from the nearby trigeminal tract, the facial, vagal, and glossopharyngeal nerve fibers innervating the middle and external ears. Thus activity of the DCN is influenced by stimulation of somatosensory system (nonauditory structures) that has been also implicated in development of tinnitus. There is a correlation with limbic structures that has been clearly documented with anxiety, depression and negative psychological state and increased tinnitus ${ }^{61}$ The ability of some individuals to modulate tinnitus by performing voluntary somatosensory or motor actions (forceful head and neck contractions) is probably attributable to somato-sensory-auditory interactions within the central nervous system of these patients which account for somatic modulation of tinnitus of Pathophysiology of limbic and autonomic nervous systems: Involvement of limbic and autonomic nervous system could explain why some people suffer from tinnitus while others do not. Approxmately $80 \%$ of tinnitus patients for the first time do not associate the sound with any negative meaning and experience spontaneous habituation. However, if the first perception of tinnitus induces high levels of annoyance by association with periods of stress and anxiety, tinnitus might lead to high levels of anxiety, resulting in enhanced activity in the limbic and autonomic nervous systems. In such situations, tinnitus emerges as a clinically significant problem. Long time noise exposure might bring about changes in the central auditory system as well as affect limbic regions such as the amygdala and hippocampus. The amygdala plays a central role to modulate auditory cortex plasticity. Tinnitus, typically induced by hearing loss, is often associated with emotional stress, depression and anatomical changes of the hippocampus. Therefore, the limbic system might play a major role in the generation as well as the suppression of tinnitus, functional magnetic resonance imaging (fMRI) also revealed moderate hyperactivity in the primary and posterior auditory cortices and limbic networks in patients with tinnitus. The greatest degree of hyperactivity observed in these areas more specifically to sounds frequency-matched to patients' tinnitus. On the other hand, few complementary structural differences were also identified in prefrontal cortex and other limbic structure. However, a strong positive corelation is also evident between two limbic regions, primary auditory cortex and tinnitus which revealed auditory limbic interaction

\section{DISCUSSION}

It appears that etiology of tinnitus is linked to several factors including hearing loss, noise, age, psychological status, ototoxicity, medical problem such as vestibular schwanoma, multiple sclerosis, somatosensory damage. The pathophysiology of tinnitus is associated with cochlea, auditory and vestibular nerve, dorsal cochlear neucleus, auditory cortex, pathology in limbic and autonomous nervous system. This systematic review is an attempt to find evidence to support these causes and pathophysiology for further tinnitus management. The studies reported differ based on sample size, study design, selection of control and experimental group, methodology, and instruments used. The authors explore the differences in studies to explain how evidence is stronger to accept the different causes and pathology underlying tinnitus generation and that influence it. According to, systematic reviews formulate research questions that are broad in scope, and identify and synthesize studies that directly relate to the systematic review question. It uses PRISMA guidelines to collect secondary data, and combine findings qualitatively or quantitatively. Systematic review studies can be rejected based on research methodology and adequate sample size is important in an effective studys otherwise, small sample sizes may limit generalization of the findings. In this systemaic review, sample size was wide ranging: Twelve studies had between 9 to 50 participants, three studies had 50 to 100 participants, three studies had 150 to 450 , and one study had 14178 participants while only one study had 75764 participants. Randomized control trial (RCT), meta-analysis, cohort study, case control study, case series are important for evaluation of clinical findings. There were only three randomized 
control trials, two experimental studies, twelve cross section analytic trials and five case control studies. In this review, the most widely used tests for finding aetiology and pathophysiology were PTA, IA, SRT, SDS, TEOAE, DPOAE,ABR, MLR, TEN, fMRI, MRI, PET, 40HzASSR, MEG, 148 channel magnetometer (4D- neuroimaging) rTMS, PET and tinnitus pitch and loudness measurement. Therefore, based on these findings it can be drawn that a reduction in cochlear activity due to aetiology such as noise, age and hearing loss, leads to changes the neural activity across several areas of the central auditory system and non-auditory canters including limbic system and the cerebellum associated with the perception of tinnitus. Thus for assessment of tinnitus PTA, IA, OAE, ABR, fMRI and $P E T$ tests are required. In a systematic review, level of evidence is an important parameter to explore strength of the study. It was possible to rate level of evidence for 30 studies in this systematic review. To interpret the level of evidence, guidelines developed by Ackley, Swan, Ladwig, and Tucker (2008) were used. It is clear that only three studies have level-I- meta analysis of randomised control trial, 25 studies have level III- well-designed controlled trials and three studies have level-IV- well-designed case-control or cohort studies to find out the aetiology and pathophysiology of tinnitus. Also these findings suggest that multidisciplinary professionals such as ENT, audiologist, radiologist and psychologist are required for a combined approach for better tinnitus assessment in future. It is possible to bring out standard normative clinical practice guidelines for tinnitus assessment based on systematic reviews of more RCTs of good quality that have similar results. This systematic review explores the many etiologic causes of tinnitus that have been proposed; but for the majority of patients with tinnitus, the etiology remains idiopathic. An extensive range of disorders can cause damage to the auditory system, potentially leading to the development of tinnitus. The major causative factors are: 1) viral infection; 2) vascular impairment; 3) immune-mediated mechanisms; 4) inner ear abnormality; and (5) CNS abnormalities, including tumors, trauma, hemorrhage, infarction, and other pathologies. The most common suspected etiologies of tinnitus in adult patients is idiopathic $(71.0 \%)$, infections $(12.8 \%)$, otologic disease $(4.7 \%)$, trauma $(4.2 \%)$, vascular or hematologic $(2.8 \%)$, neoplastic (2.3\%), and other causes $(2.2 \%)$ as potential suspected etiologies. Multiple pathophysiology were identified, including $\mathrm{OHC}$ and IHC pathology, auditory nerve synchronisation central nervous system anomalies and limbic and autonomous nervous system (problems. Establishment of a direct causal link between tinnitus and these etiologies remains elusive. Diagnostic imaging (fMRI, PET) is a useful method for identification of temporal bone or intracranial pathology that can present with tinnitus as a primary symptom.

\section{CONCLUSION}

A detailed case history and physical examination of patients with tinnitus may identify some causes, for example, trauma, cerebrovascular accident and ear surgery. MRI scanning of the internal auditory canal and cerebellopontine angle is required in patients suffering from tinnitus where the cause cannot be identified by case history or physical examination in order to rule out cases of vestibular schwannoma. Evidence suggests that tinnitus involves neuroplastic changes in central auditory structures which takes place when the brain is deprived of its normal input due to cochlear pathology. Cochlear pathology may not be identified in audiograms in some cases but can be detected with the help of OAE or ABR measures. Neural changes may occur at the level of synapses between inner hair cells and the auditory nerve or at in any level of the central auditory pathway. Presence of tinnitus for a very long time/duration usually is the results of functioning of complex network structure including central auditory and non-auditory system.

\section{CONFLICT OF INTEREST}

The authors declares no conflict of interest

\section{ACKNOWLEDGEMENT}

The authors sincerely acknowledge the contribution of Dr. Geetha Mukundan and Prof Geeta Gore, for their immense support and guidance.

\section{REFERENCES}

1. Armstrong R, Hall BJ, Doyle J, Waters E. "Cochrane Update. 'Scoping the scope' of a cochrane review". Journal of Public Health. 2011;33:147-50.

2. Bhatt JM, Lin HW, Bhattacharyya N. Prevalence, severity, exposures, and treatment patterns of tinnitus in the United States. JAMA Otolaryngology-Head \& Neck Surgery. 2016;142: 959-965.

3. Bauer CA, Brozoski TJ, Myers K. Primary afferent dendrite degeneration as a cause of tinnitus. Journal of Neuroscience Research. 2009;85:1489-98.

4. Baizer JS, Manohar S, Paolone NA, Weinstock N, Salvi RJ. Understanding tinnitus: The dorsal cochlear nucleus, organization and plasticity. Brain Research. 2012;1485:40-53.

5. Baldo P, Doree C, Molin P. Antidepressants for patients with tinnitus. Cohrane Database Syst Rev. 2009;1:1-6.

6. Bankstahl US, Elkin EP, Gebauer A, Görtelmeyer R. Validation of the THI-12 questionnaire for international use in assessing tinnitus: A multi-centre, prospective, observational study. International Journal of Audiology. 2009;51:671-677.

7. Bauch CD, Lynn SG, Williams DEMellon, MW, Weaver, AL. Tinnitus impact: Three different measurement tools. Journal of the American Academy of Audiology. 2003;14: 584-92.

8. Bogo R, Farah A, Karlsson KK, Pedersen NL, Svartengren M, Skjönsberg, Å. Prevalence, incidence proportion, and heritability for tinnitus: a longitudinal twin study. Ear and Hearing. 2003; 38: 292-300.

9. Bellini JL, Rumrill Jr PD. Research in rehabilitation counseling: A guide to design, methodology, and utilization. Charles C Thomas Publisher. Springfield. 2009;1:6-9.

10. Chang, H, Chen K, Kaltenbach JA, Zhang J, Godfrey, DA. Effects of acoustic trauma on dorsal cochlear nucleus neuron activity in slices. Hearing Research. 2004;164:59-68. 
11. Chen YC, Zhang J, Li XW, Xia W, Feng X, Gao B. Aberrant spontaneous brain activity in chronic tinnitus patients revealed by resting-state functional MRI. Neurolmage: Clinical. 2004;6:222-8.

12. Chen GD. Fechter LD. The relationship between noiseinduced hearing loss and hair cell loss in rats. Hear Res. 2003;1:81-90.

13. Chrbolka P, Paluch Z, Alušík Š. Current perspectives of tinnitus and its therapeutic options. European Geriatric Medicine. 2005;6:170-4.

14. Daniell WE, Swan SS. McDaniel MM, Stebbins JG, Seixas NS, Morgan MS. Noise exposure and hearing conservation practices in an industry with high incidence of workers' compensation claims for hearing loss. American Journal of Industrial Medicine. 2009; 42:309-317.

15. Dehmel, S, Cui YL, Shore SE. Cross-modal interactions of auditory and somatic inputs in the brainstem and midbrain and their imbalance in tinnitus and deafness. American Journal of Audiology. 2009;1:1-6.

16. Dong, S, Rodger J, Mulders WH, Robertson D. Tonotopic changes in GABA receptor expression in guinea pig inferior colliculus after partial unilateral hearing loss. Brain Research. 2010;1342:24-32.

17. Emmerich E, Richter F, Reinhold U, Linss V, Linss W. Effects of industrial noise exposure on distortion product otoacoustic emissions (DPOAEs) and hair cell loss of the cochlea long term experiments in awake guinea pigs. Hear Res. 2010;148:9-17.

18. Flor H, Elbert T, Knecht S, Wienbruch C, Pantev C, Birbaumers N, Taub E. Phantom-limb pain as a perceptual correlate of cortical reorganization following arm amputation. Nature. 2010;375:4-82.

19. Gay LR, Mills GE, Airasian PW. Educational research: Competencies for analysis and application. Boston: Pearson Education. 2011;1:1-8.

20. Geocze L, Mucci S, Abranches D, de Marco, MA, de Oliveira Penido N. Systematic review on the evidences of an association between tinnitus and depression. Brazilian Journal of otorhinolaryngology. 2011; 79:106-111.

21. Han BI, Lee HW, Kim TY, Lim JS, Shin KS. Tinnitus: Characteristics, causes, mechanisms, and treatments. Journal of Clinical Neurology. 2009;5:11-19.

22. Henry JA. Audiologic Assessment. In: Snow J. Tinnitus: Theory and management. Ontario: BC Decker Inc. 2004;1:220-36.

23. Henry JA, Dennis KC, Schechter MA. General review of tinnitus. Journal of Speech, Language, and Hearing research. 2006;1:1-6.

24. Henry JA, Roberts LE, Caspary DM, Theodoroff SM, Salvi RJ. Underlying mechanisms of tinnitus: Review and clinical implications. Journal of the American Academy of Audiology. 2014; 25: 5-22.

25. Hobson J, Chisholm E, El Refaie, A. Sound therapy (masking) in the management of tinnitus in adults. Cochrane Database of Systematic Reviews. 2011;1:1-11.

26. Holt AG, Bissig D, Mirza N, Rajah G, Berkowitz B. Evidence of key tinnitus-related brain regions documented by a unique combination of manganese-enhanced MRI and acoustic startle reflex testing. PloS one. 2010;5:14-260.
27. Jastreboff PJ, Jastreboff MM. Tinnitus retraining therapy. In: Snow J. Tinnitus: Theory and management. Ontario: BC Decker Inc. 2014;1: 295-309.

28. Jastreboff PJ, Hazell JW. Tinnitus Retraining Therapy. New York: Cambridge University Press. 2004.

29. Jastreboff PJ. The neurophysiological model of tinnitus. In: Snow J. Tinnitus: Theory and management. Ontario: BC Decker Inc. 2004;1:96-107.

30. Job A, Raynal M, Kossowski M. Susceptibility to tinnitus revealed at $2 \mathrm{kHz}$ range by bilateral lower DPOAEs in normal hearing subjects with noise exposure. Audiology and Neurotology. 2003;12:137-144.

31. Kaltenbach JA. Tinnitus: models and mechanisms. Hearing research. 2002;6: 52-60.

32. Kaltenbach JA, Zhang J, Finlayson P. Tinnitus as a plastic phenomenon and its possible neural underpinnings in the dorsal cochlear nucleus. Hear Res. 2002;6:200-26.

33. Kaltenbach JA, Zhang J, Afman CE. Plasticity of spontaneous neural activity in the dorsal cochlear nucleus after intense sound exposure. Hear Res. 2003;147:282-92.

34. Koehler SD, Pradhan S, Manis PB, Shore SE. Somatosensory inputs modify auditory spike timing in dorsal cochlear nucleus principal cells. European Journal of Neuroscience. 2011;33:409-20.

35. Koester M, Storck C, Zorowka P. Tinnitus--classification, causes, diagnosis, treatment and prognosis. MMW Fortschritte der Medizin. 2014;146:23-4.

36. Kraus KS, Canlon B. Neuronal connectivity and interactions between the auditory and limbic systems: Effects of noise and tinnitus. Hearing Research. 2014;88:34-46.

37. Kujawa SG, Liberman MC. Adding insult to injury: Cochlear nerve degeneration after "temporary" noise-induced hearing loss. Journal of Neuroscience. 2019; 29:14077-85.

38. Lanting CP, De Kleine E, Van Dijk, P. Neural activity underlying tinnitus generation: Results from PET and fMRI. Hearing Research. 2009;255:1-13.

39. Leaver AM, Renier L, Chevillet MA, Morgan S, Kim HJ, Rauschecker JP. Dysregulation of limbic and auditory networks in tinnitus. Neuron. 2009;69:33-43.

40. Levine RA, Abel M, Cheng $H$. CNS somatosensory-auditory interactions elicit or modulate tinnitus. Experimental Brain Research. 2009;153:643-48.

41. Levin RA. Tinnitus: Theory and management. Somatic tinnitus. In: Snow, J. B. editor. Ontario: BC Decker Inc. 2008;1:108-124.

42. Lockwood AH, Salvi RJ, Burkard RF, Galantowicz PJ, Coad ML, Wack DS. Neuroanatomy of tinnitus. Scand Audiol Suppl.1990;51:47-52.

43. Lockwood AH, Salv RJ, Burkard RF. Tinnitus. N Engl J Med. 2002;347:904-10.

44. López-González, MA, Cambil E, Abrante A, López-Fernández $\mathrm{R}$, Barea E, Esteban F. Tinnitus measurement with conventional audiometer versus high-frequency audiometer. Acta Otorrinolaringologica (English Edition). 2003;63: 102-105.

45. McCormack A, Edmondson-Jones M, Somerset S, Hall D. A systematic review of the reporting of tinnitus prevalence and severity. Hearing research. 2010;337:70-9. 
46. Mirz F, Gjedde A, Sdkilde-Jrgensen H, Pedersen C. Functional brain imaging of tinnitus-like perception induced by aversive auditory stimuli. Neuroreport. 2010;11:633-7.

47. Møller AR, Langguth B, DeRidder D, Kleinjung T. Textbook of tinnitus. Springer Science \& Business Media. 2010.

48. Mühlnickel W, Elbert T, Taub E, Flor H. Reorganization of auditory cortex in tinnitus. Proceedings of the National Academy of Sciences. 2010; 95:10340-43.

49. Nuttal AL, Meikle MB, Trune DR. Peripheral process involved in tinnitus. In: Snow JB. Tinnitus: Theory and management. Ontario: BC Decker Inc. 2004; 52-68.

50. Parra,LC, Pearlmutter BA. Illusory percepts from auditory adaptation. J Acoust Soc Am. 2007;121:1632-41.

51. Reyes SA, Salvi RJ, Burkard RF, Coad ML, Wack DS. Galantowicz, PJ, et al. Brain imaging of the effects of lidocaine on tinnitus. Hear Res. 2002;171:43-50.

52. Rauschecker JP, Leaver AM, Mühlau M. Tuning out the noise: limbic-auditory interactions in tinnitus. Neuron. 2007;66:819-26.

53. Salvi RJ, Wang J, Ding D. Auditory plasticity and hyperactivity following cochlear damage. Hear Res. 2007;147:26174.
54. Samarei R, Fatholahi N. Causes of tinnitus in patients referred to ENT clinic of Imam Khomeini hospital in Urmia 2012-2013. Global Journal of Health Science. 2004;6:136-8.

55. Schreiner CE, Cheung SW. Cortical plasticity and tinnitus. In: Snow JB. Tinnitus: Theory and management. Ontario: BC Decker Inc. 2004;1:189-204.

56. Shargorodsky J, Curhan GC, Farwell WR. Prevalence and characteristics of tinnitus among US adults. The American Journal of Medicine. 2004; 123: 711-18.

57. Shore SE. Plasticity of somatosensory inputs to the cochlear nucleus-implications for tinnitus. Hearing Research. $2011 ; 281: 38-46$.

58. Shore S, Zhou J, Koehler S. Neural mechanisms underlying somatic tinnitus. Prog Brain Res. 2007;166:107-23.

59. Tang J, Jii B, Liu L. Study of hearing loss in 200 patients with subjective tinnitus. Lin-Bed Journal of Otorhinolaryngology Head and Neck Surgery. 2005; 25: 726-29.

60. Wienbruch C, Paul I, Weisz N, Elbert T, Roberts LE. Frequency organization of the $40-\mathrm{Hz}$ auditory steady-state response in normal hearing and in tinnitus. Neuroimage. 2006;33:180-94.

61. Yildirim, G, Berkiten G, Kuzdere M, Ugras H. High frequency audiometry in patients presenting with tinnitus. The Journal of International Advanced Otology. 2006;6: 401-6. 